\title{
Medical Image of the Week: Atypical Deep Sulcus Sign
}

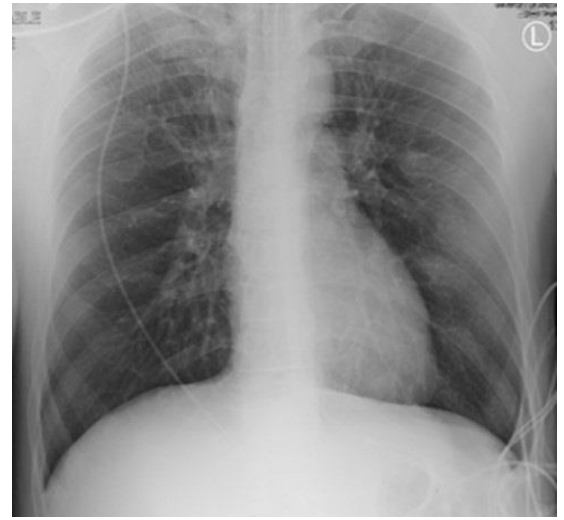

Figure 1. Chest X-ray showing hyper inflated lungs with no clear evidence of pneumothorax.

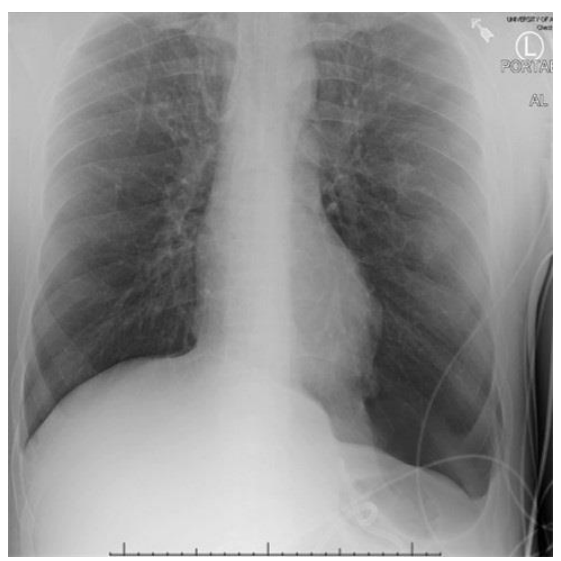

Figure 2. Atypical deep sulcus sign on the left side.

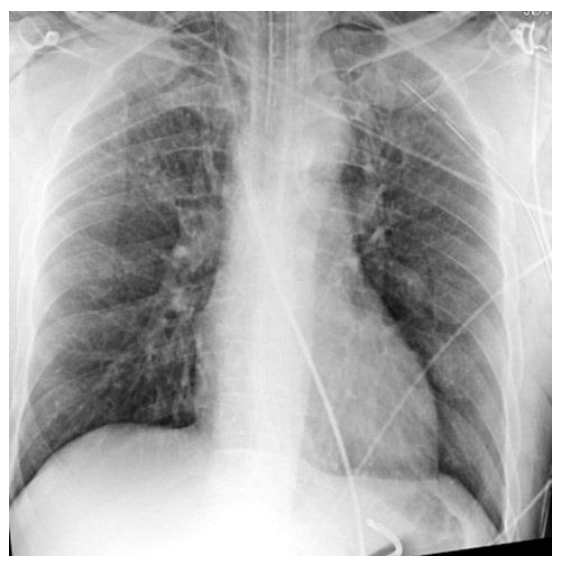

Figure 3. Complete resolution of left sided pneumothorax after chest tube placement. 
The deep sulcus sign is a radiolucent lateral sulcus where the chest wall meets the diaphragm. The costophrenic angle is abnormally deepened when the pleural air collects laterally, producing the deep sulcus sign (1). Here, we present a 42-year-old man with a history of asthma who was admitted with status asthmaticus requiring intubation. On hospital day 3, the patient developed subcutaneous emphysema surrounding his entire neck and extending into left side of the chest wall. Chest X-ray after this episode showed an atypical deep sulcus sign (Figure 2) concerning for left sided pneumothorax that was also confirmed by bedside ultrasound. A surgical chest tube was placed immediately and a repeat chest X-ray (Figure 3 ) showed complete resolution of the pneumothorax and the deep sulcus sign. In critically ill patients where it is difficult to obtain an upright film, it is important to pay attention to the costophrenic angles when concern for pneumothorax arises. In a supine film, a deep sulcus sign may be the only indication of a pneumothorax because air collects anteriorly and basally within the nondependent portions of the pleural space, as opposed to the apex when the patient is upright (2).

Hasan Ali MD¹, Huthayfa Ateeli MBBS², Bhupinder Natt MD FACP ${ }^{2}$, Sachin Chaudhary $\mathrm{MD}^{2}$

${ }^{1}$ Department of Medicine and ${ }^{2}$ Division of Pulmonary, Critical Care, Sleep and Allergy University of Arizona College of Medicine

Tucson, AZ USA

\section{References}

1. Kim HK, Park CY, Cho HM. Deep sulcus sign. Trauma Image \& Procedure. 2016;1(1):12-3. [CrossRef]

2. Liu SY, Tsai IT, Yang PJ. Pneumothorax and deep sulcus sign. QJM. 2016;109(9):621-2. [CrossRef] [PubMed] 